\title{
Peut-On Se Passer Du Concept D'ecosysteme ? Partie I : Questions Epistemologiques Et Methodologiques
}

\author{
Thierry Rolland \\ Aix Marseille Univ, CNRS, Centre Gilles Gaston Granger, \\ Aix-en-Provence, France
}

Doi:10.19044/esj.2018.v14n32p306 URL:http://dx.doi.org/10.19044/esj.2018.v14n32p306

\begin{abstract}
The concept of ecosystem played a central role in the structuring of ecology as a full-fledged scientific discipline during the twentieth century. However, certain theoretical and practical issues related to the notions of homogeneity and stability on one side and dynamic equilibrium on the other led, from an artefactual a priori, to exclude relevant phenomena from the field of analysis, such as maintaining ecosystems subject to conditions of permanent change. In this first article, after a historical and epistemological analysis of the heuristic value and the limits of the concept of ecosystem, some tracks are proposed in the direction of its maintenance by removing the ambiguities that accompany it since its origins.
\end{abstract}

Keywords: Ecosystem, stability, heterogeneity, anthropic influence, evolutionary change.

\section{Résumé}

Le concept d'écosystème a joué un rôle central dans la structuration de l'écologie comme discipline scientifique de plein exercice au cours du XXe siècle. Cependant, certains enjeux théoriques et pratiques liés aux notions d'homogénéité et de stabilité d'un côté et d'équilibre dynamique de l'autre, ont conduit, à partir d'un a priori artéfactuel, à exclure des phénomènes pertinents du champ de l'analyse, comme celui du maintien des écosystèmes soumis à des conditions de changement permanent. Dans ce premier article, au terme d'une analyse historique et épistémologique de la valeur heuristique et des limites du concept d'écosystème, quelques pistes sont proposées en direction de son maintien moyennant une levée des ambiguïtés qui l'accompagnent depuis ses origines.

Mots clés : Ecosystème, stabilité, hétérogénéité, influence anthropique, changement évolutif 


\section{Introduction}

La notion d'écosystème est aujourd'hui largement utilisée à des fins opératoires et s'est vue en grande partie vidée de sa substance théorique initiale. En effet, si l'on adopte une perspective héritée de l'histoire et de l'épistémologie des sciences, cette notion d'écosystème correspond en réalité à un travail de théorisation fruit d'un ensemble d'échanges entre scientifiques qui s'inscrit dans l'histoire de l'écologie depuis le XIXe siècle. Vocable introduit en 1935 (Tansley, 1935), la genèse du concept d'écosystème a pris racine au carrefour de plusieurs disciplines des sciences de la nature (Drouin, 1984) et a joué depuis les années 1950 un rôle unificateur dans les études écologiques, agrégeant à un niveau d'intégration en surplomb des données sur la végétation, les populations animales et le milieu physique, démontrant ainsi l'interdépendance de ces différents niveaux de vie dans la nature. Comme après lui l'étude de l'ADN qui a permis de concevoir l'unité du monde vivant, comme aujourd'hui le principe de sélection naturelle considéré par les sciences évolutives comme l'armature nomologique de l'explication de la dynamique de l'histoire de vie, l'écosystème par sa situation originale dans les sciences écologiques pose des problèmes de nature scientifique et épistémologique particuliers. La stabilisation théorique du concept après son « officialisation » dans des ouvrages de référence au-delà de la seconde guerre mondiale a conduit à une multiplication d'études internationales qui lui sont associées et qui vont faire de cet ensemble théorique un concept mobilisable et opératoire. Toutefois, le concept d'origine a subi un ensemble de réductions qui a fait passer la théorisation au second plan et a conduit à une profusion de débats sur sa valeur, certains voulant assurer sa défense (Knight, 1981), d'autres clarifier les abus et l'usage du terme (Rossiter, 1990); (O'Neill, 2001).

Il s'agit donc ici d'affirmer l'historicité du concept d'écosystème et de discuter des problèmes théoriques et scientifiques qu'il soulève et d'analyser en quoi il conviendrait de le réhabiliter dans le champ de l'écologie, voire d'élaborer un concept de substitution. Une telle démarche nécessiterait alors de désigner la sphère théorique la plus adaptée à cette réorientation conceptuelle. A partir d'une esquisse historique et d'une analyse épistémologique de la genèse de cet « objet » paradigmatique qui s'inscrit luimême dans l'histoire de la constitution de l'écologie comme science, je propose de montrer le rôle central qu'a tenu ce concept dans les débats et les enjeux théoriques qui ont mobilisé des conceptions épistémologiques souvent opposées, mais aussi parfois complémentaires qui ont finalement accompagné les différentes étapes d'édification de ce concept. Les différentes questions de nature scientifique et épistémologique soulevées par son usage seront ensuite abordées dans une deuxième partie. Enfin, la question du maintien de ce concept central ou de son remplacement conduira en conclusion à s'interroger sur les principes qui pourraient jeter les bases de son renouvellement ou de 
l'émergence d'un ou de concept(s) de substitution en écologie dans le contexte actuel du paysage évolutif généralisé des sciences du vivant.

\section{Partie I : Historicité du concept}

Si la création du mot « écosystème » est le fait d'un botaniste (A. G. Tansley) en 1935, le concept sous-jacent procède de plusieurs courants (Drouin, 1984) qui s'inscrivent dans l'histoire de l'écologie depuis le début du XIX ${ }^{\mathrm{e}}$ siècle. L'écologie s'est développée sur la base d'une tradition naturaliste descriptive, mais dès ses débuts, son essor est rythmé par l'élaboration de concepts et méthodes qui ont pour but d'expliquer la répartition et la dynamique du vivant et qui lui ont permis de se démarquer des différentes disciplines de la biologie. Pour en saisir toute la portée, la distinction entre l'écologie et les autres sciences biologiques est plus évidente en examinant les différents sujets qu'elle est amenée à traiter (Voute, 1968). Schématiquement, l'objectif principal de l'écologie fondamentale est de comprendre les causes de la diversité spécifique et les raisons de sa variation dans différentes communautés. Ces sujets impliquent non seulement l'étude des relations entre les organismes vivants et leur milieu physico-chimique (désigné par compartiment abiotique), mais également celles développées entre les organismes eux-mêmes (relations biotiques). L'expérience acquise au cours de ces travaux amène les écologues à ajuster en continu leurs concepts, entraînant ainsi une évolution rapide de l'écologie dans la deuxième moitié du XXe siècle.

Si le terme d'écosystème a été énoncé pour la première fois dans un article consacré à l'écologie en 1935, c'est le limnologiste R. L. Lindemann qui en 1942 lui donne le statut de concept (Linderman, 1942). Les études écosystémiques deviennent des analyses de flux d'énergie et de matériaux au travers d'unités composées de communautés biotiques arbitrairement définies. L'écologie des systèmes aquatiques joue un rôle de précurseur dans plusieurs domaines théoriques. Les travaux dirigés par Hutchinson, à l'origine du passage de la méthode descriptive à la méthode expérimentale en limnologie, en sont un bon exemple (Hutchinson, 1948). L'hydrobiologie passe aussi par une période « d'hégémonie » (Barnaud, et al., 1991) au moment des recherches sur l'eutrophisation des systèmes lentiques (lacs, réservoirs). Forbes, avant eux, avait décrit le lac nordique comme un microcosme, un système relativement fermé et autorégulateur (Forbes, 1925). A partir d'un inventaire des différentes espèces du lac et d'une description approfondie de sa situation géographique, Forbes développait une conception du lac comme celle d'un ensemble structuré d'éléments en interdépendance, qui tenait lieu d'approche écosystémique avant la lettre.

La généralisation de ce type d'approche est assurée par l'édition d'ouvrages de synthèse, comme le Fundamentals of Ecology (Odum, 1953), 
véritable bible des écologistes, qui officialise l'utilisation du terme écosystème en exposant les méthodes quantitatives énergétiques et en proposant différents modèles (Margalef, 1968). L'analyse des systèmes peut être vue sous-jacente à la définition d'un écosystème donnée par E. P. Odum en 1953 « comme unité naturelle qui comprend des parties vivantes et non vivantes interagissant pour produire un système stable dans lequel l'échange de matériaux entre les parties vivantes et non vivantes suit des chemins circulaires. » Elle aborde en effet les systèmes complexes comme des composants interconnectés avec des boucles de rétroaction (Hutchinson, 1948) qui stabilisent le système à un point d'équilibre relativement constant. A partir des années 60 , la généralisation des études écosystémiques devient la règle dans les programmes de recherche écologique. Inhérente à l'analyse des systèmes, l'analogie de la machine et le rôle grandissant de l'ordinateur dans les modèles écosystémiques ont permis à la science moderne des écosystèmes de développer une approche pratique de l'extrême complexité des systèmes naturels (O’Neill, 2001). Selon Drouin, «dès l'origine, les débats théoriques auxquels donne lieu le concept d'écosystème mettent en jeu une série de rapports entre l'homme et la nature, le vivant et le non vivant, l'unité et la multiplicité ». Cette analyse est confortée par P. Acot, philosophe des sciences, pour qui naît alors une "pensée écosystémique » induisant « l'apparition d'une idéologie écologiste systémiste qui transformera les représentations sociales des relations nature-société, dans les sociétés industrielles (Acot, 1994)».

De nombreux auteurs reconnaissent au concept d'écosystème un rôle fondamental en écologie. Il a permis de consolider les bases théoriques de la discipline et de donner à l'écologie, une unité d'étude autour d'un concept qui a pris une valeur paradigmatique pendant plus de 50 ans. L'écosystème est un concept parce qu'il constitue une structure intellectuelle a priori, une manière spécifique de poser un regard sur la nature. Au sens de Kuhn, il tient lieu de paradigme parce qu'il met l'accent sur certaines propriétés tout en négligeant voire en ignorant certaines autres et présente « [...] la capacité de susciter [...] des perspectives de recherche élargie sur toutes sortes de problèmes à résoudre » (Kuhn, 1962). Son rôle a été déterminant dans la mise en cohérence des principaux savoirs qui constituent l'architecture de l'écologie scientifique contemporaine, bien que le maintien de ce statut soit contesté aujourd'hui. Rétrospectivement, c'est autour de trois approches qui correspondent aux différentes orientations prises par la discipline que s'est dessinée son émergence au carrefour de plusieurs courants scientifiques : réflexions sur la répartition des organismes vivants, réflexions sur les interactions entre facteurs biotiques et abiotiques, et réflexions sur la dynamique des populations. Ces trois approches sont le reflet de conceptions épistémologiques qui traduisent à leur manière des moments de l'histoire des relations entre les sciences naturelles, les autres sciences biologiques et les sciences physiques en écologie 
depuis plus d'un siècle. Je décrirai donc ces trois approches en abordant successivement une démarche organiciste fondée sur la reconnaissance d'une spécificité de la complexité des phénomènes biologiques, une autre mécaniste en lien avec le développement de modèles mathématiques rendant compte de la dynamique des populations et en position intermédiaire une démarche systémique qui associe des phénomènes physiques et biologiques dans une théorie scientifique générale commune. Ce ne sont pas en soi différentes figures de préfiguration annonciatrices du concept d'écosystème, mais elles peuvent utilement servir à identifier les pôles du débat autour de la construction de la structure théorique du concept d'écosystème.

La démarche organiciste. C'est historiquement la démarche pour laquelle le poids des disciplines naturalistes a été le plus significatif. La géobotanique du XIXe de Humboldt (1769-1859) à de Candolle (1778-1841), Vesque (1848-1895), Bonnier (1853-1922) et Flahaut (1852-1935), avait permis d'appréhender la végétation autour de grands ensembles cohérents soumis aux éléments extérieurs, dont le climat au premier chef. Héritière de ce mouvement, l'écologie végétale naissante de la fin du XIXe de Warming (1841-1924) et Schimper (1808-1880) et des premières décades du XXe siècle va se démarquer de la dimension géographique et botanique et contribuer à théoriser des niveaux d'intégration supérieurs à l'individu et aux populations correspondant aux groupements d'espèces et aux communautés biotiques : «ce qui caractérise précisément l'évolution de la pensée pré-écologique puis écologique, c'est la subordination graduelle, à partir de la définition de la formation végétale de Grisebach, du point de vue autécologique au point de vue synécologique » (Acot, 1983). Sur le plan méthodologique, l'inventaire de groupements floristiques ou phytotaxons censés décrire des ensembles de végétation cède le pas devant l'utilisation de caractères analytiques, comme le degré d'abondance et le pourcentage de recouvrement, permettant d'appréhender la végétation à des échelles diverses, correspondant aux associations, groupements, communautés et peuplements. Cette évolution conceptuelle sera portée par l'apparition de la sociologie végétale au début du $\mathrm{XXe}$, dont on peut distinguer deux grandes écoles réparties des deux côtés de l'Atlantique. L'école européenne ou école de Zürich-Montpellier développera une conception phytosociologique de la végétation élaborée par BraunBlanquet et ses élèves. L'association végétale y est décrite comme "un groupement végétal de composition floristique déterminée, présentant une physionomie uniforme, croissant dans des conditions stationnelles uniformes et possédant une ou plusieurs espèces caractéristiques » (Braun, et al., 1913). Acot décrit une écologie végétale européenne statique (encore vivace aujourd'hui par ses orientations méthodologiques ${ }^{17}$ ) fondée sur des

${ }^{17}$ La syntaxonomie de Braun-Blanquet, basée sur le recours à la définition d'aires minima uniformes composées d'espèces caractéristiques, est encore d'usage très courant aujourd'hui, 
discontinuités de végétation comme figées par un « instantané photographique (Acot, 1994). Le recours à une métaphore similaire s'applique dès lors à une deuxième école qui se développera outre-Atlantique, celle d'une écologie dynamique qui voit la végétation comme « prise dans un mouvement cinématographique ». C'est Clements au cours des années 1930 qui développera de l'autre côté de l'Atlantique, cette écologie dynamique fondée sur la théorie des successions et le concept de climax, qui représente la formation végétale fondamentale vers laquelle tous les stades intermédiaires convergent. Clements a recours à une analogie avec le processus des phases successives de développement d'un organisme, d'où le nom de conception « organiciste » qui lui a été donné (Clements, 1936). Cette conception a imprégné de nombreux écologues qui travaillaient sur les communautés végétales et animales, qui en ont déduit des propriétés homéostatiques des milieux (Barnaud, et al., 1991). Par sa tendance à la stabilisation vers un stade ultime, au moins sur le long terme, elle suppose admis l'équilibre de la nature, axiome abandonné aujourd'hui et contribuant à la remise en cause actuelle du concept d'écosystème.

La démarche systémiste. En opposition avec cette conception de « superorganisme » portée par Clements, Tansley dans son fameux article, « The Use and Abuse of vegetational Concepts and Terms » se propose de clarifier le fondement logique d'une théorie de la végétation et introduit le vocable « écosystème ». A partir du concept de système, il développe une conception nouvellement intégratrice, qui prend en compte sur un même plan l'ensemble des éléments en interrelation le composant, qu'il s'agisse du compartiment biotique ou des facteurs abiotiques. Mais la mise en avant des facteurs inorganiques sera interprétée par les successeurs de Tansley, comme le fondement théorique d'une approche méthodologique, qui conduira à la réduction des écosystèmes à ces facteurs, ce qui traduirait un écosystème «porteur d'un caractère janussien, qui lui permettra ultérieurement d'appartenir à des perspectives épistémologiques radicalement contradictoires » (Bergandi, 1999). Ce trait janussien se retrouve chez Lindeman, membre de l'équipe G.E. Hutchinson. Il formalisera le concept d'écosystème, à partir de l'analyse des processus en jeu dans un système lacustre, composé de sous-systèmes interreliés (Egerton, 1983). Ces travaux marquent l'acte de naissance d'une théorie quantitative de l'écosystème, qui constituera le cadre théorique dans lequel s'inscriront une grande part des études écologiques des décennies qui suivront. L'analyse écosystémique a depuis cette période permis l'intégration dans l'écologie, de principes issus de différentes théories comme ceux de la cybernétique et de la communication, introduits par Margalef. Il convient également de ne pas sous-estimer dans le

en témoigne le nombre d'entrées bibliographiques dans des articles d'écologie récents supérieures à 7000 . 
développement de cette théorie, l'importance des travaux sur les cycles de la matière que Vernadski (1863-1945) appellera au XXe siècle «les cycles biogéochimiques ». En effet, de Lavoisier (1743-1794) au XVIII ${ }^{\mathrm{e}}$ siècle à Saussure (1767-1845), de Boussaingault (1801-1887) à Liebig (1803-1873), des progrès considérables ont été accomplis dans la compréhension globale des cycles d'éléments de base de la chimie du vivant. Or, ces aspects, qualifiés aujourd'hui de facteurs abiotiques, se révèleront déterminants pour expliquer la complexité à l'œuvre des mécanismes du vivant et contribueront à alimenter une certaine vision réductionniste ${ }^{18} \mathrm{~d}$ 'une écologie fondée sur une orientation

18 Selon le réductionnisme, pour n'importe quelle discipline scientifique, l'ensemble des lois qu'elle présente (les axiomes et donc aussi les théorèmes) peut en principe être explicitement déduit à partir de lois d'une science plus fondamentale. Ayala définit, selon le type d'entités concerné, trois types de réduction : ontologique, méthodologique et épistémique (Ayala, et al., 1974). Selon le réductionnisme ontologique, la dernière réalité d'un quelconque niveau d'une certaine entité ou classe d'entités (par exemple, un organisme) est constituée par, et réductible à, un autre genre d'entités (par exemple, les molécules) discrètes du niveau inférieur à celui considéré. Le réductionnisme méthodologique soutient l'idée que les propriétés d'une entité ou niveau d'intégration donné peuvent être prévues et/ou expliquées à partir d'interactions entre ses composants : les bilans énergétiques issus de la théorie quantitative de l'écosystème de Lindeman en sont une illustration. Enfin, le réductionnisme épistémique considère que la connaissance que l'on a dans un certain domaine (par exemple, sur les organismes) peut être réduite à la connaissance que l'on a dans un autre domaine (par exemple, sur les molécules et leurs interactions), ou (à la manière de Nagel) que les lois théoriques relatives à la biologie peuvent être remplacées par des lois à des niveaux plus fondamentaux, comme celles de la biophysique ou de la physico-chimie. Parmi les exemples classiques de réductions, mentionnons la réduction des gènes à des segments d'ADN. Presque tous les biologistes sont aujourd'hui physicalistes au sens où ils considèrent que les êtres vivants sont composés uniquement de parties matérielles. Néanmoins, la thèse réductionniste selon laquelle les théories biologiques sont en principe réductibles à des théories de sciences plus fondamentales et en particulier à la physique et la chimie, est controversée. A mesure que l'on découvre les détails du mécanisme extrêmement complexe de l'expression des gènes, il apparait désormais comme une simplification abusive d'identifier un gène à un segment d'ADN (Morange, M., 1998). Morange souligne notamment que sans la hiérarchie organique constituée de différents niveaux d'organisation qui permet l'expression des gènes, le génome en lui-même ne serait rien d'autre qu'une mémoire morte (Morange, 2002). Ce pouvoir d'action propre des gènes est à la base du postulat fondateur de la génétique qui voit dans l'état moléculaire d'un système une détermination de son état macroscopique selon la chaîne de causalités : gènes - protéines - phénotype. Ce déterminisme adopté par les biologistes moléculaires du XXe siècle tient beaucoup à l'analyse qu'en fit Erwin Schrödinger (Schrödinger, 1944), notamment pour la physique, qu'il voyait soumis à un "principe d'ordre à partir du désordre ", alors que la biologie de son côté devait répondre à un « principe d'ordre à partir de l'ordre ». Ce principe d'ordre correspond bien sûr à l'information génétique qui serait aux commandes des mécanismes physiologiques de manière strictement déterministe, sans quoi la variabilité de ces mécanismes serait trop importante et incompatible avec la précision qui les caractérise. Or, ce principe, très critiqué sur le plan théorique, est invalidé, d'une part par l'absence d'infaillibilité (des erreurs se produisent tout au long de la chaine « gène-protéine ») et d'autre part, par les nombreuses données expérimentales récentes, notamment celles qui portent sur le manque de spécificité des protéines (Albert, 2005), la plasticité des sites d'interaction moléculaire (Ma, et al., 2011) ou encore la structure désordonnée des protéines (Dunker, et al., 2000). Sans rentrer dans le détail des résultats qu'elles présentent, elles suggèrent en effet que c'est l'état macroscopique (le phénotype) à travers les structures cellulaires qui contraint les protéines à interagir de manière spécifique. On se retrouve donc avec des chaînes de réaction moléculaire qui, au lieu d'être la cause des processus cellulaires, en seraient plutôt le résultat, et donc devant un « paralogisme finaliste classique » qui consiste à inverser la cause et l'effet, ce qui constitue « une contradiction totale du déterminisme génétique » (Kupiec, 2010). Toutefois, selon le regretté Jean Gayon, si on laisse de côté les manifestations les plus caricaturales dont il est parfois fait usage (les gènes « commandent le caractère » par exemple), comme 
physico-chimique de l'écosystème, de Hutchinson à Odum. L'intégration des lois de la thermodynamique et de la conservation de masses dans la science moderne des écosystèmes a mis en lumière le caractère paradoxal du statut actuel de l'écosystème, porté par un holisme ${ }^{19}$ conceptuel, mais décliné dans des méthodologies analytico-réductives, ce qui aux yeux de certains révèlerait une relative inconsistance du concept, traduirait pour d'autres une des failles à l'origine de l'instabilité du concept et plus généralement de l'écologie (Cherret, 1990). La focalisation des études sur la circulation, la transformation, et l'accumulation d'énergie et de matière par l'intermédiaire des êtres vivants et de leurs activités, les digressions actuelles de la recherche écosystémique vers un certain réductionnisme, rendraient ainsi la discipline incohérente (Schlesinger, 1989). En revanche, d'autres considèrent que les écosystèmes sont à la fois «le résultat observable et la cause formelle » des principaux phénomènes écologiques, et donc pleinement intégrés aux principes les plus établis de l'écologie (Burns, 1990).

La démarche mécaniste. Ce mouvement est considéré comme la grande période de l'écologie théorique, en particulier dans les années 1920-1930, par l'introduction de modèles mathématiques dans l'étude des populations (Lotka, 1925) ; (Volterra, et al., 1935). Cette étape a été importante car elle a permis, en testant les hypothèses des statisticiens, de favoriser la pénétration progressive à partir des années 1960 de la dimension évolutive des populations par le prisme de la régulation des populations (Delord, 2010). Mac Arthur en

l'ont fait remarquer de nombreux philosophes des sciences (par exemple Nagel 1961, ou Popper 1972), la réductibilité d'une théorie scientifique, entendue au sens de sa déductibilité à partir d'une autre théorie, marque un progrès dans l'unification des connaissances et dans la compréhension des phénomènes (Gayon, 1995). Notre connaissance n'est point appauvrie, mais authentiquement approfondie, lorsque par exemple nous savons déduire (et éventuellement rectifier) les lois du mouvement des planètes de Kepler à partir de la mécanique newtonienne.

${ }^{19}$ Selon le «holisme », doctrine proposée par Smuts, homme politique sud-africain, dans les années 1920, une explication adéquate d'un phénomène doit tenir compte de la totalité du système (holisme vient de holos, qui signifie « tout » en grec ancien) qui est à l'origine de ce dernier et non pas se focaliser sur l'une de ses parties, dans la mesure où le " tout » est irréductible à chacune de ses parties. Pour Aristote, si un tout vivant est plus que l'ensemble de ses parties, si une main coupée du corps n'est plus une main, le tout inerte ou passif de la machine ne sera qu'un « agrégat », un ensemble de parties. Claude Bernard considère que « Le physiologiste et le médecin ne doivent jamais oublier que l'être vivant forme un organisme et une individualité [...]. Il faut donc bien savoir que si l'on décompose l'organisme vivant en isolant ses diverses parties, ce n'est que pour la facilité de l'analyse expérimentale et non point pour les concevoir séparément » (Bernard 1865, II, ch. ii, § 1). Si l'on reprend l'exemple du réductionnisme génétique, le niveau génétique ne peut pas endosser le statut d'explanans visant à expliquer un explanandum hors de portée, qui ne peut pas y être réduit ; la conception holiste considère que l'ensemble (d'un génome, d'un organisme, d'un écosystème) ne peut pas être réduit à la somme des entités qui le composent. Formulé différemment, il est en principe impossible d'expliquer ou de prédire un phénomène biologique sur la base d'un explanans qui contiendrait uniquement les mécanismes d'expression des gènes. Une explication adéquate de ce phénomène supposerait de faire appel à un système plus vaste qui inclut ces mécanismes génétiques, ainsi que d'autres éléments, variables et relations, irréductibles à ces mêmes mécanismes. Voilà ce que serait alors une posture holiste, doctrine selon laquelle une explication adéquate doit tenir compte de la totalité du système qui est à l'origine d'un phénomène et non pas se focaliser sur l'une de ses parties, dans la mesure où le « tout » est irréductible à chacune de ses parties. 
1967 introduit les notions de « stratégie démographique » (Mac Arthur, et al., 1967) soumise à la sélection de traits démographiques, par la suite considérablement affinées par l'étude de l'ensemble des traits d'histoire de vie (taille, nombre de descendants, ...) (Stearns, 1977). La progression de ce courant s'est faite successivement, tout d'abord par l'étude des fluctuations densitaires (ou cinétique démographique), puis par celle des facteurs à l'origine de ces fluctuations et enfin par l'étude de la biologie des populations, fondée sur une approche pluridisciplinaire, incluant tout aussi bien la génétique et les mathématiques que la biochimie, la physiologie et l'écologie (Legay, et al., 1985). La mobilisation de l'outil mathématique et de l'outil expérimental a permis la recherche de relations de cause à effet dans l'étude des phénomènes en biologie des populations. Ancrée dans un relatif réductionnisme, cette approche exerce un attrait certain chez les biologistes d'hier comme d'aujourd'hui (Barnaud, et al., 1991). Elle témoigne d'un passage d'une écologie descriptive à un stade théorique fondé sur des problématiques intégrant la dimension évolutive qui requiert une prise en compte de l'espace et de son hétérogénéité, ainsi que du changement vu comme élément moteur du complexe « population-environnement ». Avant les années 1950 cependant, les écologues de leur côté n'estimaient pas que leur discipline relève de considérations évolutives. Même si ces divergences se sont depuis amenuisées, à travers une structuration démographique des populations en classes, en écologie des populations notamment, le fossé conceptuel qui en résulte avec l'écologie écosystémique, fondée sur des conceptions d'équilibre et d'homogénéité d'espace est encore aujourd'hui très profond.

La distinction entre ces différents courants peut paraitre schématique, mais elle traduit bien les obstacles qui ont animé la discipline et la confrontation entre des conceptions méthodologique et épistémologique, qui ont permis des va-et-vient conceptuels, dont le concept d'écosystème constitue le point d'orgue en quelque sorte. Les débats imposés par l'évaluation classique et traditionnelle des sciences, notamment entre holisme et méthodologie intégrative d'une part et réductionnisme et méthodologie analytico-réductive d'autre part, ont nourri cette confrontation mais ont également contribué à l'émergence du concept. Ces oppositions entre méthodes descriptives et mathématiques (Roughgarden, et al., 1989), entre approches réductionnistes et holistiques (Friederichs, 1958), vont persister tout au long du XXe siècle et alimenter une réticence de plus en plus grande vis-àvis du statut paradigmatique de l'écosystème, quand « on songe (...) au regard complexe des écologues contemporains, à la fois engagés dans la production de modèles mathématiques, dans la saisie du réel par réduction au physicochimique, et également soucieux de ne pas s'éloigner de la tradition naturaliste (Acot, 1994). » L'alternative entre des modes d'explication « réductionnistes » et « holistiques » demeurera ici hors de notre propos. Mais, partant du constat 
que l'élaboration du concept d'écosystème a été d'inspiration multiple, nous nous demanderons en quel sens elle s'est trouvée confrontée dans sa structure théorique "d'équilibre dynamique » aux problèmes théoriques posés par le changement généralisé du contexte évolutif contemporain.

\section{Partie II : Questions épistémologiques et problèmes scientifiques}

Questions épistémologiques

Une fois postulées la place centrale du concept d'écosystème en écologie et sa fécondité à faire émerger de nouvelles idées pour résoudre les problèmes d'environnement sensu lato, ses limites après un demi-siècle d'application sont aujourd'hui de plus en plus apparentes. Les critiques les plus fréquentes portent sur la valeur du concept. Les dérives de son emploi relèvent de la confusion entre le modèle, outil destiné à faciliter l'étude, et l'objet réel analysé. Pour certains, elles conduisent à élaborer des modèles quantitatifs parfois éloignés de la réalité à partir d'unités d'étude écosystémiques supposées homogènes (forêt, étangs) afin d'assurer une large portée aux résultats (Blandin, et al., 1989). Pour d'autres, l'écosystème n'est qu'une affirmation sur les contraintes physiques qui pèsent sur les êtres vivants, un cadrage spatio-temporel étroit limité par notre capacité à englober une réalité plus large (Sagoff, 1997). Il est alors considéré comme un spécimen abstrait sans lieu ni histoire, excluant de fait l'homme. Cette interrogation sur la délimitation exacte des contours de l'entité « écosystème » semble légitime dans la mesure où la spatialité qu'il est censé incarner, nécessairement empirique, est une recherche constante de sa congruence avec les systèmes physiques réels. Comme le souligne Bergandi, l'histoire des sciences naturelles a nourri des scénarii ontologiques qui « sont constitués d'entités à la fois imaginaires, complexes et heuristiques [...] car il s'agit de constructions de l'esprit qui, seules, coïncident rarement avec des entités naturelles douées d'une valeur concrète de réalité (Bergandi, 2007). » L'adéquation entre la représentation mentale et l'entité physique réelle semble alors le plus souvent résulter d'un accommodement avec les besoins de la recherche (Blandin, 2007). D'un point de vue méthodologique, l'écosystème est représenté par une entité locale délimitée spatialement, mais le réseau d'interactions dans lequel cette entité se trouve est le plus souvent négligé, voire non pris en compte, alors que les interactions avec l'extérieur sont déterminantes pour le renouvellement et le maintien de ses différentes composantes. Cette orientation méthodologique est ancrée dans une conception fortement influencée par les problématiques de protection de la nature (Blandin, et al., 1984). Dans ce contexte, l'identité écosystémique est déterminée par la représentation d'un système en équilibre, pour lequel la question est moins de savoir comment il évolue mais plutôt comment et quand il est susceptible de retrouver son équilibre. L'équilibre est alors un donné ontologique, à la manière des entités 
chez Aristote soumises ontologiquement au repos qui est leur condition première : «[...] Ce repos immuable de l'être pleinement réalisé [...]» (Koyré, 1986). Tout se passe comme si l'état anormal issu d'un désordre ne pouvait que déboucher sur un retour, après dissipation du désordre, de l'antééquilibre. Le temps est alors considéré comme une donnée « reproductible », dans la mesure où les phénomènes temporels se répèteraient d'un cycle à l'autre, d'une succession à l'autre. Cette conception du temps rejoint celle de la physique newtonienne, dont les lois sont invariantes par rapport à la direction du temps (Delord, 2010). Une temporalité abstraite baigne dès lors la théorie des écosystèmes qui pour l'essentiel considère et analyse les phénomènes complexes d'interactions, de flux de matière et d'énergie avec des outils épistémologiques de la physique fondamentale. L'équilibre ou le retour à l'équilibre de l'entité écosystème est alors assuré par les relations dynamiques des différents éléments qui entrent dans sa composition et en permanence en arrangement fonctionnel et en coordination les uns avec les autres. Cette conception de type «structuraliste» a conduit nombre de contempteurs de l'écosystème à considérer qu'il ne répondait pas à la réalité écologique car sans lieu et sans histoire. Serait-ce une forme d'idéalisation d'une nature protectrice et bienfaisante, qui, malgré les assauts du quotidien, demeure inchangée et conserve sa dimension consolatrice face à notre désarroi ontologique? Il ne s'agit pas d'aborder ici par sa dimension psychologique la portée d'une telle conception, cependant, on ne peut que constater que dans une large mesure, la question de la temporalité a été négligée dans les différentes études en écologie, jusqu'à une époque récente, si ce n'est dans le cadre d'études diachroniques sur des intervalles de temps limités, censées mesurer la variation par rapport à un état référence du milieu. Cette vision « idéaliste » a imprégné la pratique des études de terrain qui impliquait de faire référence à l'écosytème-concept, fondement d'une possible généralisation : l'exemple cité par Blandin d'une forêt péri-urbaine d'un système populationsenvironnement particulier, montre que cette forêt particulière était considérée comme « un représentant d'un unique type d'écosystème, l'écosystème « forêt péri-urbaine » (les études dans une forêt A pouvaient donc servir de référence sur les conséquences d'un événement dans une forêt B) (Blandin, 1992). Aujourd'hui, l'approche pragmatiste qui conduit à reconnaître des « objets » écologiques locaux et transitoires est plutôt celle qui est favorisée. Dans ce contexte, les écologues savent que les écosystèmes qu'ils étudient sont une composante parmi d'autres dans des systèmes écologiques plus vastes, plus ou moins imbriqués et interactifs. Car l'écosystème n'est ni homogène, ni équilibré, ni homéostatique et la stabilité est un mythe hérité du fameux « équilibre de la nature » (Soule, et al., 1995). Pickett et al. résument ces différentes critiques : " Le paradigme classique de l'écologie, avec son insistance sur l'état stable, sa suggestion de systèmes naturels fermés et 
autorégulateurs, et sa résonance avec l'idée non-scientifique d'équilibre de la nature, ne peuvent plus servir comme une base adéquate pour la conservation (Pickett, et al., 1992). \) Ces ambiguïtés définitionnelles du concept rendent difficile son exploration critique et nécessitent un examen des présupposés implicites des différentes applications dont il est l'objet. Cet examen portera sur des problèmes logiques et scientifiques posés par les concepts de stabilité et d'équilibre, de non prise en compte des perturbations d'origine anthropique et du contexte évolutif contemporain.

\section{Problèmes méthodologiques et logiques.}

La conception d'un écosystème en "équilibre dynamique » a longtemps prévalu chez les chercheurs écologues. Face à la complexité des phénomènes qu'elle aborde, l'étude d'un système écologique a longtemps buté sur le manque de méthodologie applicable à l'ensemble du système, ce qui explique que les travaux aient été menés sur des parties ou des propriétés particulières du système étudié. Concrètement, l'hypothèse d'un « équilibre dynamique » va requérir trois conditions, comme on peut le voir sur la figure 1 : un espace homogène, un espace fini, un système stable. Elle correspond à une représentation où le système étudié est un espace « naturel » théorique en situation de confinement et d'isolement relatif, vis-à-vis des perturbations extérieures. L'avantage de cette démarche est de permettre de mettre en évidence des propriétés spécifiques de ces sous-ensembles. Son inconvénient est de réduire notre réflexion à quelques hypothèses qui orientent le regard vers un type de réponses attendues et qu'il convient donc d'interroger.

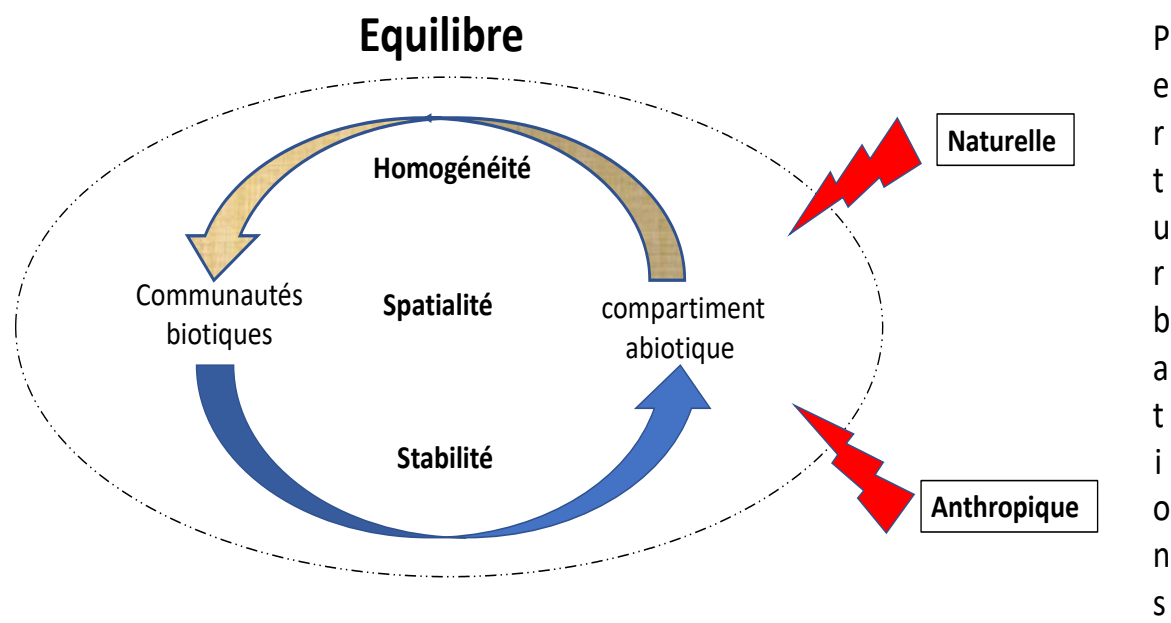

Figure .1: Représentation de l'écosystème selon l'ancienne conception héritée du mythe de " l'équilibre de la nature ». Elle est traduite ici par le tryptique Spatialité-Homogénéité-Stabilité.

Le trait discontinu vient indiquer que des entrées et sorties du système sont possibles, mais de façon limitée, si ce n'est dans les cas où des perturbations (d'origine naturelle ou anthropique) viendraient bouleverser cet équilibre de l'extérieur. 
Tryptique stabilité - spatialité - homogénéité. Classiquement, le concept d'écosystème requiert un espace spécifique, circonscrit par exemple à une parcelle de forêt en milieu terrestre ou à un étang en milieu aquatique, dans lequel des dynamiques internes pourront faire l'objet de quantification de flux de matières et d'énergie, incluant des boucles de rétroaction et d'interaction, tolérant un certain degré de substituabilité entre les organismes, à l'intérieur des limites définies par l'étude. L'identification de chaînes trophiques composées de différents compartiments (des producteurs Iaires, consommateurs Iaires, prédateurs et parasites jusqu'aux décomposeurs) a permis d'expliquer ces dynamiques internes, mais très vite, les anomalies d'un tel modèle apparaissent. La démarche classique en effet consiste à moyenner les différentes hétérogénéités au niveau local, de façon à obtenir un espace homogène où des propriétés spécifiques peuvent être étudiées. Or, l'hétérogénéité spatiale permet de maintenir toute une gamme de populations, dont des espèces pionnières nécessaires à la recolonisation du milieu après un déséquilibre. L'exemple des résurgences phréatiques qui peuvent emprunter des réseaux de noues résultant du relief naturel, comme d'anciens bras morts ou de dépressions allongées, plus ou moins connectés aux systèmes aquatiques existants, sont typiques à cet égard. Elles bénéficient de conditions relativement homogènes sur le long terme et constituent un espace refuge pour toute une série d'espèces, qui pourront après une crue par exemple, recoloniser le milieu. Ne pas tenir compte de la dynamique de ces systèmes particuliers conduit à négliger les potentiels de régénération de l'écosystème, dans les diagnostics qui sont opérés. Pour comprendre le fonctionnement d'un lac ou d'une rivière, les hydrobiologistes prennent en compte l'importance des transferts d'éléments allochtones, produits essentiellement par les écosystèmes terrestres. Le critère d'homogénéité correspond de fait à un écosystème intrinsèquement instable et rendu plus sensible au changement en réduisant sa capacité au retour à l'équilibre, même si ce nouvel équilibre diffère du précédent. L'intérêt de l'analyse de l'hétérogénéité spatiale dans la structuration et l'organisation des systèmes écologiques est donc une nécessité et « la stabilité d'un système écologique ne peut être prédite par une théorie qui ignore l'hétérogénéité (O'Neill, 2001). " Cette homogénéité contraint la recherche d'une spatialité confinée à des limites spécifiées sur le terrain d'étude. C'est dans ces limites que les différentes distributions spatiales des populations sont étudiées. D'un point de vue pratique, la délimitation du champ de l'étude est une nécessité, mais le plus souvent au prix d'une structure isolée abstraitement des espaces en contiguïté avec elle. Car ces limites empiriques peuvent être débordées par certaines populations. Les exemples ne manquent pas de populations, animales ou végétales, dont l'aire de répartition est bien plus étendue que les limites spécifiées. Des transferts de population hors système peuvent changer la donne au niveau local, déplaçant l'équilibre vers 
d'autres populations susceptibles d'exercer leur influence sur l'ensemble du milieu étudié. Un mouvement inverse vers les limites extérieures du système peut engendrer des situations critiques pour la stabilité de l'écosystème. La modification des conditions physico-chimiques locales (température moyenne en hausse avec des extrêmes amplifiés, pluviométrie modifiée...) est une des causes potentielles de cette instabilité. Elle exerce un effet attracteur pour des espèces mieux adaptées et répulsif pour d'autres plus sensibles qui vont alors migrer hors système. La non prise en compte des échanges inter-groupements et inter-systèmes gomme les effets d'interface et de bordure aux limites de l'écosystème. Or, négliger ces échanges, c'est minimiser la substituabilité implicite des espèces dans une certaine proportion, essentielle au maintien des fonctions de la structure trophique du milieu dans son rôle de recyclage et circulation des nutriments. Si une liste d'espèces définit un écosystème, alors l'écosystème n'est plus le même après modification et récupération de l'équilibre sur la base d'une liste différente. Le présupposé de stabilité est donc indissociable d'une prise en compte des espaces autour des limites définies et d'une certaine substituabilité des espèces pour assurer les fonctions d'échange, de recyclabilité et d'élimination au sein des écosystèmes.

Influence anthropique. «L'exclusion de l'homme est, pour l'écologie, dès qu'elle se fait écosystémique, une nécessité de structure (Larrère, 1991) ». Ainsi l'homme serait extérieur à l'écosystème et ne participerait en rien à la dynamique des écosystèmes, si ce n'est pour détourner des biens et services à son profit. Son influence est alors considérée comme perturbatrice et assimilée à un «bouleversement» des 《équilibres préexistants ». Cette restriction est souvent présentée comme un accommodement à une modélisation du système, mais elle limite la portée des résultats (Barnaud, et al., 1991). La nature des questions posées par la gestion et l'aménagement de l'espace en est un bon exemple. Sans prise en compte de l'hétérogénéité spatiale (la fragmentation des habitats est un enjeu pour les biocénoses des écosystèmes concernés) et des changements liés aux activités humaines, les enjeux cruciaux sur des sujets tels que la préservation de la biodiversité et la durabilité des milieux ne peuvent être que minimisés et insuffisamment traités. La transformation de l'environnement physique s'est faite à un tel degré que la structure écologique des écosystèmes (productivité, composition taxonomique) a profondément été modifiée. Dans de nombreux cas, le maintien de la diversité biologique nécessite une gestion à l'échelle biogéographique ou à celle du paysage dépassant les intérêts locaux. La bonne échelle d'étude doit alors faciliter la compréhension des mécanismes en jeu. Par la « respatialisation » des systèmes écologiques, elle permet la prise en compte de l'hétérogénéité, ainsi que l'intégration de l'homme comme composante et moteur de l'évolution des systèmes écologiques (Lefeuvre, et al., 1988). Ce qui conduit à la nécessité pour les écologues d'intégrer la dimension historique de ces changements dans 
leurs analyses structurales et fonctionnelles. Cette évolution des idées ouvre ainsi la voie vers une coopération interdisciplinaire entre sciences biologiques et sciences humaines (Turner, 1989).

Contexte évolutif. Une population d'individus est impliquée en permanence dans un réseau complexe d'interactions avec d'autres populations sous l'influence réciproque des conditions physico-chimiques d'un biotope : « la rigueur de ces interactions laisse à penser l'importance qu'aura toute variation de ces écosystèmes sur l'évolution de chacune des espèces qui les composent (Lamotte, et al., 1985). " La pression anthropique de nature chimique ou physique sur les milieux augmente les contraintes sur ces populations conduisant à des ajustements permanents, incluant des remplacements d'individus voire une substitution de population par une autre facilitée par l'opportunité de niches écologiques laissées vacantes. Conceptuellement, les substitutions d'espèces se produisent en vertu d'une stabilité recherchée et d'un équilibre à retrouver. Comment dès lors concevoir l'action de la sélection naturelle dans un tel contexte ? C'est probablement une des limitations ou contradictions majeures du concept actuel d'écosystème. Les populations par le jeu des interactions qui les anime au sein d'un écosystème ont été façonnées par la sélection naturelle. Elles sont le résultat d'une collection d'évènements dont elles permettent de retracer l'histoire. D'une part, la sélection naturelle a conduit à l'obtention de populations qui sont supposées avoir optimisé leur potentiel biotique aux taux fixés par les conditions physico-chimiques du milieu. Mais d'autre part, elle est censée fonctionner sur des temps relativement longs, incompatibles avec le maintien de la stabilité du milieu sur de telles échelles temporelles. Comment alors penser la substitution de certaines espèces en lieu et place d'autres en cas de perturbation du système, sur un temps court, si ce n'est dans un cadre conceptuel renouvelé qui prend en compte les changements liés aux activités humaines et les conditions de non-équilibre aux échelles locales et globales des écosystèmes.

\section{Conclusion}

L'écosystème, concept central dans la jeune écologie du $\mathrm{XX}^{\mathrm{e}}$ siècle, peine à rendre compte de situations concrètes sur le terrain dans la nouvelle perspective de changement évolutif, qui constitue le contexte actuel de l'écologie et de l'ensemble des disciplines de la biologie. Originellement défini comme structure en équilibre dynamique, donc susceptible de bénéficier d'un retour à l'équilibre, seule la dimension spatiale était et est encore prise en compte dans les études de terrain. L'écosystème était et reste an-historique. Le temps n'a pas de prise sur lui et se réduit à une question de délai - délai nécessaire pour le retour à une normalité, c'est-à-dire pour que l'écart à la norme causé par des perturbations se réduise. L'écosystème s'est mué alors en 
un simple cadrage spatio-temporel, dont l'usage abusif dans de nombreux champs disciplinaires en témoigne à l'évidence. Alors, peut-on s'en passer ? Probablement pas. Ces critiques ne sont pas nouvelles. Mais elles rendent nécessaire un examen attentif des limites du concept afin de proposer quelques pistes en direction du maintien du concept moyennant une levée des ambiguïtés qui l'accompagnent depuis ses origines.

Une redéfinition autour des notions d'hétérogénéité spatiale, d'intégration de l'influence anthropique et de prise en compte de la dimension temporelle permettrait de le dépoussiérer et ainsi de le conserver. C'est notre regard sur les phénomènes naturels qui imprime sa marque sur les conceptions héritées de la théorisation de nos observations empiriques. Les critères de stabilité et d'homogénéité qui structurent le concept actuel d'écosystème favorisent un état d'esprit qui exclut des phénomènes pertinents, comme celui du maintien des écosystèmes soumis à des conditions de changement constant. Les activités anthropiques sont la cause majeure de ce changement, et l'étude des milieux doit prendre en compte leur capacité à changer d'état en réponse à un spectre continu de variabilité. Sur le plan fondamental, l'intégration du rôle moteur, à la fois structurant et déstructurant, des processus anthropiques dans la dynamique des milieux et des peuplements qui les composent, permet une compréhension des systèmes écologiques complexes comprenant 1'homme ; elle aboutit, au plan finalisé, à la gestion des milieux et des espèces. Elle a donc des implications très concrètes dans le domaine de la biologie de la conservation.

Réinscrit dans un processus de changement permanent, « réhistoricisé », la prise en compte de variabilité dans l'espace et dans le temps au-delà des limites d'un système écologique local permettrait d'englober les processus et contraintes qui pèsent sur la stabilité et la durabilité de l'écosystème. Un système local, dont le potentiel environnemental est optimisé, est exposé à l'instabilité si le potentiel change. Le défi est donc de comprendre comment un système se maintient à travers toute une série d'ajustements adaptatifs dans un cadre de changement permanent. Le maintien ou la « survie » d'un écosystème ne s'accompagne pas nécessairement d'une maximalisation de son potentiel, car ce dernier peut bénéficier d'une amélioration de sa persistance par une diminution de sa biomasse ou encore par une réduction du succès reproductif de ses composantes. Si la dynamique de l'écosystème est déterminée par les mécanismes internes des boucles de rétro-action et leur interaction avec la sélection naturelle, la prise en compte des activités anthropiques, la rapidité des adaptations ou désadaptations dans des milieux soumis à des pressions constantes, rendent difficile la prévision de son évolution dans le temps. Comme tous les systèmes écologiques, les écosystèmes sont confrontés à des problèmes de survie. Quels sont les mécanismes fonctionnels propres qui leur permettent de se maintenir tout en 
se transformant? C'est à partir de ce questionnement que sera abordé dans un deuxième article à venir le thème de l'évolution des systèmes d'organisation supérieurs en écologie.

\section{References:}

1. Acot, P. 1983. Darwin et l'écologie. Revue d'histoire des sciences, Vol. Tome 36, n¹. 1983, pp. 33-48.

2. Acot, P. 1994. Histoire de l'Écologie. Paris : PUF, 1994.

3. Albert, R. 2005. Scale-free networks in cell biology. Journal of Cell Science.2005, Vol.118, pp.4947-4957.@

4. Ayala, F. J. et Dobzhanski, T. 1974. Studies in the Philosophy of Biology: Reduction and Related Problems. s.l. : Ed. Francisco Ayala and Theodosius Dobzhansky, University of California Press, Berkeley and Los Angeles, Introduction viii, ix, 1974.@

5. Barnaud, G. et Lefeuvre, J.C. 1991. L'écologie, avec ou sans l'homme ? Jollivet, Marcel (dir.). Sciences de la nature, sciences de la société : Les passeurs de frontières. Nouvelle édition [en ligne]. Paris : CNRS Éditions. 1991, pp.69-112.틈

6. Bergandi, D. 1999. Les métamorphoses de l'organicisme en écologie : De la communauté végétale aux écosystèmes. Revue d'histoire des sciences, tome 52, n¹.1999, pp.5-21.@

7. Bergandi. 2007. Niveaux d'organisation : évolution, écologie et transaction. [éd.] Coordonné par T. Martin et al. In Le tout et les parties dans les systèmes naturels. Coll. Philosophie des sciences. s.1. : [éd.] Coordonné par T. Martin et al. In Le tout et les parties dans les systèmes naturels. Coll. Philosophie des sciences. s.1. : Vuibert, 2007. pp. 4755.@

8. Blandin, P. 1992. De l'écosystème à l'écocomplexe. [éd.] Entre nature et Société, les passeurs de frontière Jollivet. 1992, pp. 267-279. @

9. Blandin, P. et Lamotte, M. 1985. Ecologie des systèmes et aménagement : fondements théoriques et principes méthodologiques. s.l. : in Fondements rationnels de l'aménagement d'un territoire, M. Lamotte (ed.), Masson, p. 139-162., 1985.@

10. Blandin, P. et Lamotte, M. 1989. L'organisation hiérarchique des systèmes écologiques. Atti del $3^{\circ}$ Congresso Nationale della Societa Italiana di Ecologia. 1989, pp.35-48. @

11. Blandin, P. 2007. L'écosystème existe-t-il ? s.1. : Le Tout et la Partie en Ecologie. [éd.] Coordonné par T. Martin et al. In Le tout et les parties dans les systèmes naturels. Coll. Philosophie des Sciences. s.1. : Vuibert, 2007.음 
12. Braun, J. et Furrer, E. 1913. Remarques sur l'étude des groupements de plantes. Bulletin de la Société languedocienne de géographie, Montpellier, Vol. 36. 1913, p. 17.

13. Burns, T.P. 1990. The power of the ecosystem concept and paradigm. in Kawanabe, H. et al. (eds), Ecology for tomorrow. Phys. and Ecol. Japan, 27, Special number. 1990, pp. 191-197. @

14. Cherret, J.M. 1990. The contribution of ecology to our understanding of the natural world : a review of sorne key ideas. in Kawanabe, H. et al. (eds), Ecology for tomorrow. Phys. and Ecol. Japan, 27, Special number. 1990, pp. 1-16.

15. Clements, F.E. 1936. Nature and Structure of The Climax. Journal of Ecology. 1936, pp. 252-284. @

16. Delord, Julien. 2010. Ecologie et évolution : vers une articulation multi-hiérarchisée. [éd.] Philippe Huneman, Guillaume Lecointre, Marc Silberstein, Préface de Jean Gayon, Postface de Richard Lewontin sous la direction de Thomas Heams. s.l.: Collection Matériologiques, Ed. Sylepse, 2010.pp.607-628.@

17. Drouin, J.M. 1984. La naissance du concept d'écosystème. s.1. : Thèse de Doctorat de 3e cycle en Philosophie, Université Paris I, 1984.@

18. Dunker, A.K., et al. 2000. Intrinsec protein disorder in complete genomes. Genome Informatics. 2000, Vol. 11, pp.161-171.@

19. Egerton, F.N. 1983. The history of ecology : achievements and opportunities, Part one. J. Hist. Biol., 16. 1983, pp. 259-311.@

20. Forbes, S. A. 1925. The Lake as a Microcosm. 1925, pp. 537-550.@

21. Friederichs, K. 1958. A definition of ecology and sorne thoughts about basic concepts. Ecology, 39. 1958, pp.154-159.@

22. Gayon, Jean. 1995. La biologie darwinienne de l'évolution est-elle «réductionniste»? Revue Philosophique de Louvain. Quatrième série, tome 93, n¹-2,. 1995, pp. 111-139. @

23. Hutchinson, G. E. 1948. Circular causal Systems in Ecology. Annals of the New York Academy of Sciences 50. 1948, pp. 221-246. @

24. Knight, R.L. \& Swaney, D.P. 1981. «In defense of ecosystems ». Am. Nat., 117. 1981, pp.991-992.@

25. Koyré, A. 1986. Études galiléennes. s.1. : Ed. Hermann, Éditeur des sciences et des arts, 341 p., p. 20, 1986.

26. Kuhn, Thomas. 1962. La structure des révolutions scientifiques. s.1. : Ed. Flammarion, 2008, 1962.

27. Kupiec, Jean-Jacques. 2010. Une approche darwinienne de l'ontogénèse. [éd.] Syllepse. Les mondes darwiniens : l'évolution de l'évolution, sous la direction de Thomas Heams, Philippe Huneman, Guillaume Lecointre, Marc Silberstein, Préface de Jean Gayon, 
Postface de Richard Lewontin, Collect. Matériologiques. 2010. pp. 471-494.@

28. Lamotte, M. et Blandin, P. 1985. La transformation des écosystèmes cadre et moteur de l'évolution des espèces. Scientia, Vol. La Vita et la sua Storia. 1985,pp.161-190.@

29. Larrère, R. 1991. L'écologie ou le geste d'exclusion de l'homme. Paris : Maîtres et protecteurs de la nature - Champ Vallon, 1991.

30. Lefeuvre, J.C. et Barnaud, G. 1988. L'Écologie du paysage : mythe ou réalité ? Bull. Écol., 19. 1988, pp. 493-522.

31. Legay, J.M. et Debouzie, D. 1985. Introduction à une biologie des populations. Paris : Masson, 1985.

32. Linderman, R.L. 1942. "The trophic-dynamic aspect of ecology ». Ecology, 23. 1942, pp. 399-418.

33. Lotka, A. J. 1925. Principles of Physical Biology. Baltimore : s.n., 1925.

34. Ma, B, et al. 2011. Multiple diverse ligands binding at a single protein site : a matter of pre-existing populations. Protein Science. 2011, pp. 184-197.@

35. Mac Arthur, R.H. et Wilson, E.O. 1967. The Theory of Island Biogeography. Princeton : Princeton UP, 1967.@

36. Margalef, E. R. 1968. Perspectives in ecological theory. Chicago : Univ. of Chicago Press., 1968.

37. Morange, M. 1998. La part des gènes. Paris : O. Jacob. Trad. anglaise par M. Cobb, The Misunderstood Gene, Harvard University Press, 2001, 1998.

38. Morange, M. 2002. The Misunderstood Gene. s.l. : Harvard University Press, 2002.p.240. @

39. Odum, Eugène P. 1953. Fundamentals of Ecology. s.l. : 3e edition. s.l. :W.B. Saunders Company, 1953.@

40. O'Neill, V. Robert. 2001. Is it time to bury the ecosystem concept? With fully military honors, of course! Ecology. 2001, pp.3275-3284.@

41. Pickett, S. T. A., Parker, V. T. et Fiedler, P. L. 1992. The new paradigm in ecology: implications for conservation above the species level. New York: in P. L. Fiedler and Jain, editors. Conservation biology: the theory and practice of nature conservation, Chapman \& Hall, p. 65-88, 1992.@

42. Rossiter, A. ,. 1990. Ecology, environment and economics : a Pandoran Perspective. in Kawanabe, H. et al. (eds), Ecology for tomorrow. Phys. and Ecol. Japan, 27, Special number. 1990, pp. 169-189.

43. Roughgarden, J., May, R.M. et Levin, S. 1989. Perspectives in ecological theory. 1989,pp. 3-10. @ 
44. Sagoff, M. 1997. Muddle or muddle through? College of William and Mary Law Reviews, 38. 1997, pp.825-993.@

45. Schlesinger, W.H. 1989. Discussion : ecosystem structure and function. Princeton : Roughgarden, J. et al. (eds), Perspectives in ecological theory, Princeton University Press, 1989. pp. 268-274.@

46. Schrödinger, Erwin. 1944. Qu'est-ce-que la vie ? . 1993. Paris : Le Seuil, 1944.@

47. Soule, M. et Lease, G. 1995. Reinventing nature? Washington, D.C., USA : Island Press, 1995.@

48. Stearns, S.C. 1977. The evolution of life history traits: a critique of the theory and a review of the data. Annual Review of Ecology and Systematics, 8.1977,pp.145-171.@

49. Tansley, A.G. 1935. The use and abuse of vegetational concepts and terms. Ecology. 1935, pp. 284-307.@

50. Turner, M.G. 1989. Landscape ecology : the effect of pattern on process. Ann. Rev. Ecol. Syst., 20. 1989, pp.171-197.@

51. Volterra, V. et D 'Ancona, U. 1935. s.l. : Les associations biologiques au point de vue mathematique. Act. Sci. et Ind. no. 243. Paris: Hermann., 1935, Les associations biologiques au point de vue mathematique. Act. Sci. et Ind. no. 243. Paris: Hermann.

52. Voute, A.D. 1968. Ecology as a teleological science. Acta Biotheor? 1968,pp.143-164.@ 